\title{
Expert Control Based on Neural Networks for Controlling Greenhouse Environment
}

\author{
Le $\mathrm{Du}$
}

Beijing Institute of Technology, Beijing, 100081, China

\begin{abstract}
Depending upon the nonlinear feature between neural units in artificial neural networks (ANN), artificial neural network was used to develop a model for greenhouse inside air temperature management. Data was collected and processed for training and simulation of temperature model. The design of the network structure and transfer function was also discussed. Because heuristic logic of greenhouse environment exists, a simple ANN controller does not perfect complex task, the algorithm of ANN with heuristic logic is developed. Experimental result indicated that the performance of the new control scheme meet the requirement of greenhouse environment control.
\end{abstract}

Keywords: complex system, neural network, greenhouse environment.

\section{Introduction}

The greenhouse environment belongs to the complex system (G. van Straten et al., 2000; N. Sigrimis et al.,2000; M.Trigui et al., 2000), it is difficult to control due to high nonlinear, and uncertain. System configuration is shown in Fig. 1.

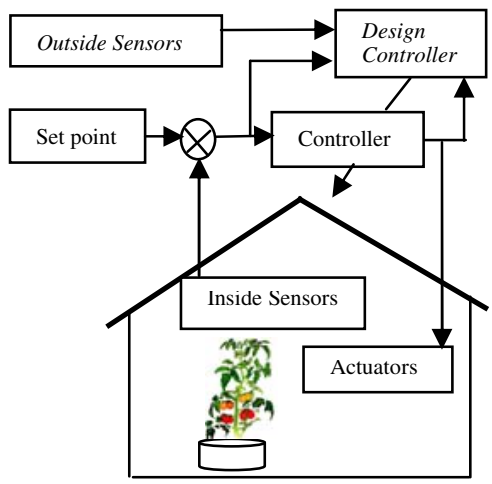

Fig. 1. Greenhouse Environment Control Architecture

The main purpose of greenhouse is to improve the environmental conditions in which plants are growth . The aim of greenhouse environmental control is to provide means to further improve these conditions in order to optimize the plant production process . The greenhouse climate is influenced by many factors, for example inside air 
temperature, the actuators, outside weather and crop. Inside air temperature is the most important factor affecting crop growth in greenhouse climate. Temperature exerted significant effects on photosynthesis. The net photosynthetic rate $(\mathrm{Pn})$ varied in day time, it was double peak curve ,there was obvious midday depression of photosynthesis, and $21-33^{\circ} \mathrm{C}$ was the suitable temperature for photosynthesis in summer. Methods aimed at efficiently controlling the greenhouse temperature must take these influences into account, and that is achieved by the use of models. This model is of capital interests in the study of the dynamics of the greenhouse temperature under different climate conditions, aimed at producing inside temperature regularity which helps to avoid extreme situations (high temperature or low temperature, etc.) and to optimize crop production by achieving adequate temperature integrals while reducing pollution and energy consumption.

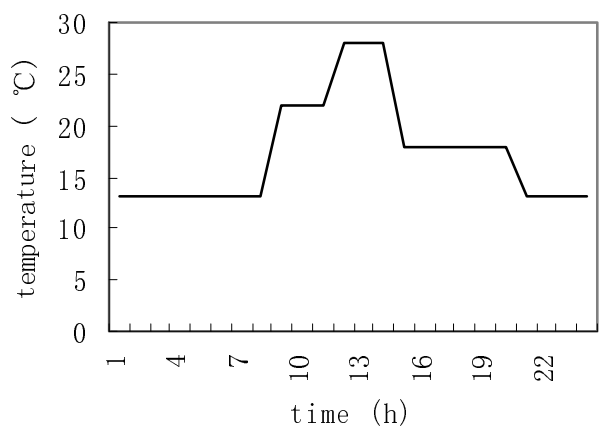

Fig. 2. Chart of variable - temperature

In this control system for plant growth, the key problems include control system input so called set point and control algorithm. The traditional set point is shown in Fig. 2. Because set point changes abruptly, that wastes a large of energy sources. So we must seek a method to transform a segment of steps chart into a smooth cure for saving energy.

If we control a variable - temperature, the traditional method is to use a controller (PID) to generate control output $\mathrm{u}(\mathrm{t})$. However, the control $\mathrm{u}(\mathrm{t})$ relates many actuators, so optimal method is used to decide what actuator to act. Since the traditional method is difficult to control this system, Artificial neural networks and symbolic description methods are introduced.

Artificial neural networks are widely applied in greenhouse environmental control to perform some type of non-linear system mapping. In the fields of identification and modeling of non-linear systems their universal approximation property is exploited. Previous work on this ANN modeling task relied in an input output model structure selected from (Cunha et al., 1996) in the context of dynamic temperature models identifications. This structure was selected by means of the (Akaike 1974) information criterion where several hypothesis were tested and the best one chosen. The application of RBF NN to greenhouse inside air temperature modeling has been investigated (Ferelra, P.M. Ruano, A.E., 2002). These models always arise like nonstability, non-regularity, which result in the complete loss of energy. This paper uses artificial neural network theory and method to control the range of temperature change and discuss the ANN model with heuristic logic. 


\section{Algorithm Design of Neural Networks Direct Self-tuning Control for Greenhouses with Heuristic Logic}

Direct self-tuning control with an artificial neural network is shown in Fig.3 which includes three basic section:

(1) Feedback loop consists of self-tuning controller and controlled plant.

(2) The parameters of the controller are obtained by designing artificial neural networks identification and controller.

(3) Heuristic logic decision what actuators are operated .

It is obvious that the key technique of self-tuning controller is how to design artificial neural networks identification and controller well and give appropriate heuristic logic.

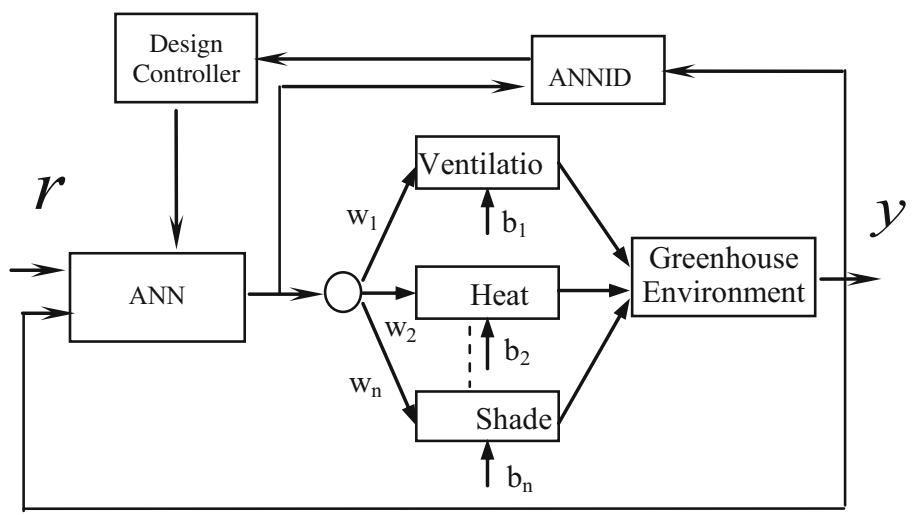

Fig. 3. Neural networks indirect self-tuning control Consider the nonlinear system, $w_{1}, w_{2}, \cdots, w_{n}$ are the weight value of $n$ actuators, $b_{1}, b_{2}, \cdots, b_{n}$ are the valve value of $n$ actuators.

$$
\begin{aligned}
& y(k+1)=g[y(k), \cdots, y(k-n+1 ; u(k), \cdots, u(k-m+1)] \\
& +\varphi[y(k), \cdots, y(k-n+1) ; u(k), \cdots, u(k-m+1)] u(k)
\end{aligned}
$$

Where $u, y$ are the input and output of the system, and $g[\bullet], \varphi[\bullet]$ are nonzero function.

If $g[\bullet], \varphi[\bullet]$ are known functions, control algorithm of the controller according to certain equivalence principle is as follow:

$$
u(k)=\frac{-g[\bullet]}{\varphi[\bullet]}+\frac{r(k+1)}{\varphi[\bullet]}
$$

The control system attempts to make the output $y(k)$ match the reference input $r(k)$. 
If $g[\bullet], \varphi[\bullet]$ are unknown functions, the artificial neural networks identification is trained on line so that $g[\bullet], \varphi[\bullet]$ approximate controlled plant. Using $N g[\bullet], N \varphi[\bullet]$ substitute for $g[\bullet], \varphi[\bullet]$, control algorithm of the controller according to certain equivalence principle is as follow:

$$
u(k)=\frac{-N g[\bullet]}{N \varphi[\bullet]}+\frac{r(k+1)}{N \varphi[\bullet]}
$$

where $N g[\bullet], N \varphi[\bullet]$ is nonlinear dynamic the artificial neural networks respectively.

\subsection{The Artificial Neural Networks Identification}

For simple problem, one order system is governed by the following equation:

$$
y(k+1)=g[y(k)]+\varphi[y(k)] u(k)
$$

The artificial neural networks identification is shown in Fig. 3. The identification consists of two three-lays nonlinear DTNN, where number of order of $N g[\bullet], N \varphi[\bullet]$ is equal to number of order of $g[\bullet], \varphi[\bullet]$. The input of the artificial neural networks is $\{y(k), u(k)\}$, and the output is as follows:

$$
\hat{y}(k+1)=N g[y(k) ; W(k)]+N \varphi[y(k) ; V(k)] u(k)
$$

where $W, V$ are two neural networks weights which are given:

$$
\begin{aligned}
& W(k)=\left[\boldsymbol{W}_{0}, W_{1}(k), w_{2}(k), \cdots, W_{2 p}(k)\right] \\
& V(k)=\left[v_{0}, v_{1}(k), v_{2}(k), \cdots, v_{2 p}(k)\right]
\end{aligned}
$$

with $p$ is hidden layer' node number

$$
w_{0}=N g[0, W] ; v_{0}=N \varphi[0, V]
$$

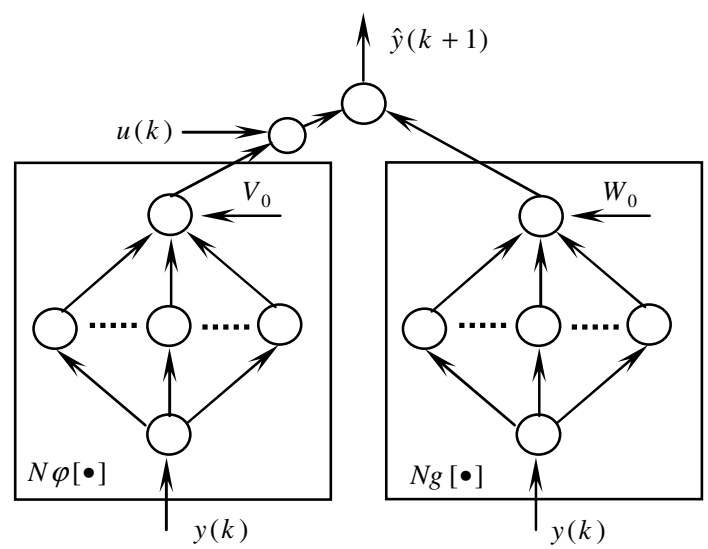

Fig. 4. The artificial neural networks identification 
In Fig. 4, $\mathrm{L}$ is linear node, and $\mathrm{H}$ is nonlinear node.

substitute (4) into (5), then the output of the control system:

$$
y(k+1)=g[y(k)]+\varphi[y(k)]\left\{\frac{-N g[\bullet]}{N \varphi[\bullet]}+\frac{r(k+1)}{N \varphi[\bullet]}\right\}
$$

if $N g[\bullet] \rightarrow g[\bullet]$ and $N \varphi[\bullet] \rightarrow \varphi[\bullet]$,

$$
y[k] \rightarrow r[k] \text { 。 }
$$

By minimizing the quadratic performance criterion, get weights tuning process thought training the artificial neural networks identification

$$
E(k)=\frac{1}{2}[r(k+1)-y(k+1)]^{2}=\frac{1}{2} e^{2}(k+1)
$$

$W(k+1)=W(k)+\Delta W(k) ; \quad V(k+1)=V(k)+\Delta V(k)$

Using Bp learning algorithm (Ferelra et al., 2002; Tetsuo et al., 2000)

$$
\Delta_{w_{i}}(k)=-\eta_{w} \frac{\partial E(k)}{\partial_{w_{i}}(k)} ; \Delta v_{i}(k)=-\eta_{v} \frac{\partial E(k)}{\partial_{v_{i}}(k)}
$$

Substitute (7) into (8), we get:

$$
\Delta_{W_{i}}(k)=-\eta_{w} \frac{\varphi[y(k)]}{N \varphi[y(k) ; V(k)]}\left\{\frac{\partial N g[y(k) ; W(k)]}{\partial W_{i}(k)}\right\} e(k+1)
$$

In the some way, we get:

$$
\Delta_{\mathcal{V}_{i}}(k)=-\eta_{v} \frac{\varphi[y(k)]}{N \varphi[y(k) ; V(k)]}\left\{\frac{\partial N \varphi[y(k) ; V(k)]}{\partial \nu_{i}(k)}\right\} e(k+1) u(k)
$$

where $\varphi[y(k)]$ is unknown and sign is known, which is marked as $\operatorname{sgn}\{\varphi[y(k)]\}$.After $\operatorname{sgn}\{\varphi[y(k)]\}$ is substituted to $\varphi[y(k)]$ of equation (9) and (10), $w_{i}(k+1), v_{i}(k+1)$ are given by

$$
\begin{aligned}
& W_{i}(k+1)=w_{i}(k)-\eta_{w} \frac{\operatorname{sgn}\{\varphi[y(k)]\}}{N \varphi[y(k) ; W(k)]}\left\{\frac{\partial N g[y(k) ; W(k)]}{\partial W_{i}(k)}\right\} e(k+1) \\
& v_{i}(k+1)=v_{i}(k)-\eta_{v} \frac{\operatorname{sgn}\{\varphi[y(k)]\}}{N \varphi[y(k) ; V(k)]}\left\{\frac{\partial N \varphi[y(k) ; V(k)]}{\partial \nu_{i}(k)}\right\} e(k+1) u(k)
\end{aligned}
$$

\subsection{Control Algorithm with Heuristic Logic}

As stated above, given control u involves many actuators operation, so control output $\mathrm{u}$ of fist step does not take action on any actuator, control output u must accomplish following mapping to yield real control output for actuator. 


$$
\begin{gathered}
u(t) \cdot \omega_{1}-b_{1}=u_{r 1} \\
u(t) \cdot \omega_{2}-b_{2}=u_{r 2} \\
\vdots \\
u(t) \cdot \omega_{n}-b_{n}=u_{r n}
\end{gathered}
$$

Where $w_{1}, w_{2}, \cdots, w_{n}$ and $b_{1}, b_{2}, \cdots, b_{n}$ result from heuristic logic, so the control algorithm possesses intelligence.

\section{Experimental Results of Control}

In this part, the effectiveness of the proposed control method will be demonstrated by a case study for winter heating system, many actuators are forbidden due to energy conservation, the actuator of heating system operates only. In this case, $w_{2}=1$, and $b_{2}$ is determined by prior knowledge. Fig 5 is experimental chart measured by the control system in Feb. 9, 2004.

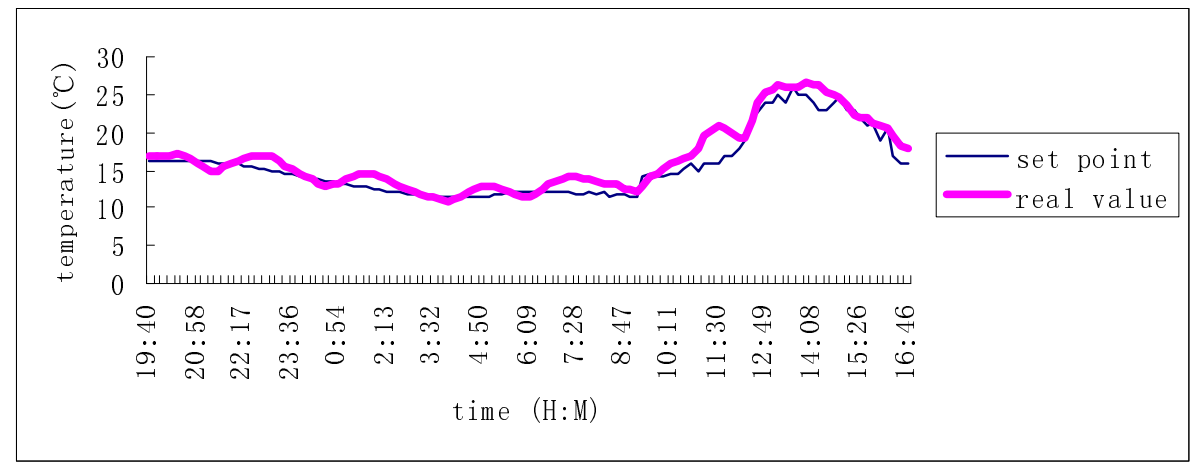

Fig. 5. Comparison chart of temperature between set point and real value

From Fig 5, it is seen that the chart of real control value tracts that of set point and light intensity increases in Fig 5 gradually at 7 to 16, provided energy for greenhouse decrease gradually. Energy chart omitted.

\section{Conclusion}

The presented the algorithm of ANN with heuristic logic proved to be very effective in meeting formal requirements for greenhouse control such as set point and tracking set point. ANN modeling set point is feasible. The key technique for greenhouse environment control uses ANN and heuristic logic reasonably to create a good algorithm. The proposed control algorithm is currently implemented in based CAN bus control system for greenhouse environment control. 


\section{References}

van Straten, G., Challa, H., Buwalda, F.: Towards user accepted optimal control of greenhouse climate. Comp. Electronics Agric. 26(3), 221-238 (2000)

Sigrimis, N., Anastasiou, A., Rerras, N.: Energy saving in greenhouses using temperature integration: a simulation survey. Comp. Electronics Agric. 26(3), 321-341 (2000)

Trigui, M., et al.: A strategy for greenhouse climate control. part I: model development. J. Agric. Engng. Res. 78(4), 407-413 (2000)

Cunha, J., Boaventura, A.E., Farria, E.A.: Dynamic temperature models of a soil-less greenhouse. In: Proceedings of the 2nd Portuguese Conference on Automatic Control, Portuguese Association of Automatic Control, Porto, Portugal, vol. 1, pp. 77-81 (1996)

Akaike, H.: A new look at the statistical model identification. IEEE transactions on Automatic Control 19, 716-723 (1974)

Ferelra, P.M., Ruano, A.E.: Choice of RBF model structure for predicting greenhouse inside air temperature. In: 15th Triennial World Confress, IFAC, Barcelona, Spain (2002)

Morimoto, T., Hashimoto, Y.: An intelligent control for greenhouse automation, oriented by the concepts of SPA and SFA - - an application to a post-harvest process. Comp. Electronics Agric. 29(2), 3-20 (2000) 